\title{
Quantitative Psychology as Mediator Between Mathematics and Psychology
}

\author{
Timo von Oertzen ${ }^{1}$
}

[1] University of the Federal Forces Munich, Neubiberg, Germany.

\begin{abstract}
Quantitative and Computational Methods in Behavioral Sciences, 2021, Article e6205,
\end{abstract} https://doi.org/10.5964/qcmb.6205

Published (VoR): 2021-05-11

Corresponding Author: Timo von Oertzen, Universität der Bundeswehr München, Fakultät für Humanwissenschaften, Institut für Psychologie, Werner-Heisenberg-Weg 39, D-85577 Neubiberg, Deutschland. Email: timo@unibw.de

It is a great pleasure to announce the publication of the inaugural issue of Quantitative and Computational Methods in the Behavioral Sciences (QCMB), a journal that is published under the Leibniz Institute for Psychology's (ZPID) PsychOpen GOLD program. The program allows to leverage professional support in editing while maintaining an open access journal without fees for either readers or authors. In the following, I would like to explain what motivated us to found this journal and why I am excited that we are going into the production stage now.

Quantitative and computational behavioral science is a very rewarding and very challenging field of science because of its location at the intersection of a behavioral and a mathematical science. In my experience from both fields, a major difference between these two is the publication strategy. In behavioral science, research is often motivated by filling a gap in the literature, choosing a topic which is neighboring existing literature while covering new ground. If we compare science to brickwork, behavioral science is building a driveway to your house: ideally, every new brick fits smoothly next to existing bricks. If necessary, little bits are chipped away from existing or new bricks to make the fit better. Sometimes bricks must also be set in completely new places. What of course rarely happens is that a brick is set on top of another brick.

In mathematical sciences, the majority of research aims at appending existing research. Articles often prove just one lemma which would be impossible to prove without a lot of previous work and which in itself often adds little value other than serving as the fundament for another brick. In the metaphor of brickwork, the typical strategy for selecting research topics in mathematics is comparable to building a wall. Note that the bricks on the lowest row usually do not contribute to the final purpose of the wall 
in total - for example, stopping wind or rain - although they are critical for the wall itself. Considering the age of mathematics, part of the wall is impressively high and would long have collapsed without the mortar of strict formalization, generalization, and simplification.

The wall and the driveway form substantially different goals, and the respective strategies, with some exceptional cases, are probably best for the respective purposes. Quantitative behavioral science finds itself at an odd combination of the two: Like substantive behavioral scientists, we have to realize the breadth of the field for which we analyze, refine or suggest methods, both to generate the right methods and also to make the field aware of most suitable methods for a given problem. This requires a constant communication with substantive fields in behavioral sciences. In my experience, it helps tremendously to maintain such steady contact with researchers that generate theories and data, a permanent and ideally balanced reciprocal learning and teaching process.

That said, if we only keep the immediate needs and applications in view, we miss out an important part of our field. It is tempting to focus on the immediate benefits and rely on applied examples: It is typically easier to publish mathematical articles if they come with a direct application, those articles will likely be cited more often, and even in teaching it is often easier to keep attention high when providing applied examples. However, examples are always a specialization of a method or mathematical theory, and thus come at the cost of generalizability. If we introduce a new method and combine it with a typical example, the reader (and sometimes even the author) may miss that the method can be applied to other questions equally well. In consequence, the same method is often introduced multiple times with multiple different notations and emphases, leading to a canon of methodological teaching that lacks generalization and simplification and is thus very tough to learn (especially for students at behavioral sciences where only limited learning resources can be allocated to methods). Scientific results that even have no immediate benefit but are only designed to combine or simplify existing methods or lay foundations for new methods are even harder to teach or to publish. The resulting situation is a classical short-term vs. long-term vicious cycle: Although short-term applied examples make teaching as well as writing articles easier, it leads to a long-term wild growth of methods that make methods harder to communicate, which in turn leads to a pressure to even more strongly rely on keeping direct touch with applications even when that is not appropriate for some scientific results.

For this reason, I am convinced that a good quantitative behavioral researcher is not only a good behavioral researcher or a good statistician (although, of course, a substantial portion of our work could as well come from either of these two groups). Beyond this, the important task that we are well suited for is to understand what part of mathematics is needed, transform this observation in mathematical assumptions and derive the lemmata, theorems or other formal conclusions that hold under these assumptions, and finally transfer the results back to the field of substantive behavioral sciences. 
In other words, where a statistician aims at most general theories, our job is to outline the sub-situation in which empirical work happens and find the most general theories in these borders. And while the behavioral scientist working on a specific problem should identify, and correctly apply, the one method that is best suited for the research topic, our job is to investigate how the same abstract situation can be found in a different concrete research question and combine existing methods into overarching frameworks that can be understood and taught compactly and easily.

Therefore, QCMB wants to provide platforms for articles in two tracks: One 'dissemination section' that provides a platform to disseminate new methods, and another 'fundamental section' that aims at theoretical results that improve our fundamental understanding of existing methods and lay the groundwork for new methods. The fundamental research section is the right one for you if you have got new formal results that advance fundamental understanding, like mathematical proofs or simulations, performance analyses of existing software, or new software or parts of it, say, a new optimization algorithm that works specifically good for Bayesian estimation in the one or other application. Other examples could be results from machine learning that can advance what we do in the behavioral sciences, or mathematical heuristics that guide which methods or study design a researcher should use for a research question at hand. In other words, things which are really beautiful, important and complex yet which otherwise you would bury in an appendix somewhere because the audience of other journals would not be interested in this aspect.

What you don't need for this section is a direct link to a specific application of your method or a real data example. Instead, you should provide theoretical (conceptual or mathematical) justification for the utility of your methodological work in future methodological or substantive research. Articles in this section are not designed to produce a large impact factor by themselves. Rather, the section aims at articles that have a deep impact. That is, these articles are likely cited by only few other articles, which themselves may become highly cited - for example, articles that forge a new method and software to apply it from the mathematical foundations presented in the original article published in the QCMB fundamental research track. Such a publication platform in our view is currently lacking, but nevertheless very important, because otherwise no fundamental derivation will ever be used in behavioral sciences unless they can be explained in a single article to an audience which has a focus on a specific application of the method.

At the same time, creating quantitative methods without ever putting them to use is like building a boat in the desert. Mathematics is arguably the most advanced science in the academic spectrum at the moment, but even die-hard natural sciences with a long-standing mathematical tradition have difficulty of linking the latest mathematical results to their research. Thus, QCMB also offers a methods dissemination section to tie the mathematical fundamental work to the data analysis problems that we encounter as 
empirical scientists. This section is for you if you are building upon thoughts published elsewhere (by you or others) to create something that substantive researchers can and should use. For example, this could be a stepwise introduction to a software tool. Tools and Techniques that are designed to be used by a broad audience, even though they build on complex mathematical results, are also well suited for this section. Such tools could for example provide help for big data analyses, or to create optimal study designs.

Articles in this section don't require to build the mathematical derivation of new methods from ground zero if it is published elsewhere already, although they should convince the reader that they can benefit from the method in their research. Especially articles introducing computational methods are not required to prove every step again when the correctness of the method can also be cited from the literature. As a reader, this section is most recommended if you are curious for the new methods around, or a new implementation of old methods, or ideally combination of existing methods, but you are not planning on extending the methods yourself or building new ones.

The editorial team of QCMB encourages methodologist to publish theoretical result and analyses even if they don't expect a large readership, but rather few readers that may build important new steps on these results, and at the same time encourage the authors of such follow-up methods, in turn, to publish explicitly with a readership in mind that can bring modern methodology to a good use in all fields of behavioral sciences.

\section{Managing Editor of Quantitative and Computational Methods in Behavioral Sciences (QCMB) \\ Timo von Oertzen}

